\title{
Non-Abelian black holes in string theory
}

\author{
Pablo A. Cano, ${ }^{a}$ Patrick Meessen, ${ }^{b}$ Tomás Ortín ${ }^{a}$ and Pedro F. Ramírez ${ }^{a}$ \\ ${ }^{a}$ Instituto de Física Teórica UAM/CSIC, \\ C/ Nicolás Cabrera, 13-15, C.U. Cantoblanco, E-28049 Madrid, Spain \\ ${ }^{b}$ HEP Theory Group, Departamento de Física, Universidad de Oviedo, \\ Avda. Calvo Sotelo s/n, E-33007 Oviedo, Spain \\ E-mail: pablo.cano@uam.es, meessenpatrick@uniovi.es, \\ Tomas.Ortin@csic.es, p.f.ramirez@csic.es
}

AbStract: We study a family of 5-dimensional non-Abelian black holes that can be obtained by adding an instanton field to the well-known D1D5W Abelian black holes. Naively, the non-Abelian fields seem to contribute to the black-hole entropy but not to the mass due to their rapid fall-off at spatial infinity. By uplifting the 5-dimensional supergravity solution to 10-dimensional Heterotic Supergravity first and then dualizing it into a Type-I Supergravity solution, we show that the non-Abelian fields are associated to D5-branes dissolved into the D9-branes (dual to the Heterotic "gauge 5-branes") and that their associated RR charge does not, in fact, contribute to the entropy, which only depends on the number of D-strings and D5 branes and the momentum along the D-strings, as in the Abelian case. These "dissolved" or "gauge" D5-branes do contribute to the mass in the expected form. The correct interpretation of the 5-dimensional charges in terms of the string-theory objects solves the non-Abelian hair puzzle, allowing for the microscopic accounting of the entropy. We discuss the validity of the solution when $\alpha^{\prime}$ corrections are taken into account.

Keywords: Black Holes in String Theory, Black Holes

ARXIV EPrint: 1704.01134 


\section{Contents}

1 5-dimensional non-Abelian black holes 2

2 Embedding in $d=10$ Heterotic Supergravity 5

$\begin{array}{lll}3 & \text { String theory interpretation } & 7\end{array}$

4 Discussion $\quad 11$

A The issue of $\alpha^{\prime}$ corrections $\quad 12$

Introduction. One of the common features of black holes or black rings with genuinely non-Abelian fields ${ }^{1}$ in Einstein-Yang-Mills (EYM) theory, where they are only known numerically [7,8], or in $\mathcal{N}=2, d=4,5$ Super-EYM (SEYM) theories [9-12], where they are known analytically, is that their non-Abelian fields fall off at spatial infinity so fast that they cannot be characterized by a conserved charge. For this reason they are sometimes called "colored" black holes, as opposed to "charged" black holes. As a consequence, the parameters that characterize the black holes must be understood as pure non-Abelian hair.

In the SEYM case it has also been observed that the non-Abelian fields seem to contribute in a non-trivial way to the BH entropy because their near-horizon behavior is similar to that of their Abelian counterparts [9-12]. Thus, apparently, the entropy of these nonAbelian black holes and rings depends on non-Abelian hair! If the BH entropy admits a microscopic interpretation, this conclusion is clearly unacceptable.

In this paper we are going to solve this puzzle for a family of particularly simple non-Abelian 5-dimensional black holes that can be embedded in String Theory [11] and which can be seen as the well-known 3-charge D1D5W black-hole solutions discussed in ref. $[13]^{2}$ with the addition of a BPST instanton [15], which is genuinely non-Abelian in the sense discussed above. ${ }^{3}$ The embedding is realized via Heterotic Supergravity (that is: $\mathcal{N}=1, d=10$ supergravity coupled to vector supermultiplets) without the terms of higher order in the curvature of the torsionful spin connection which corresponds to the

\footnotetext{
${ }^{1}$ That is: non-Abelian fields that cannot be related to an Abelian embedding via a (possibly singular) gauge transformation [1]. Gauge transformations, whether regular or singular, have no effect whatsoever on the spacetime metric and, therefore, if the non-Abelian fields can be related to an Abelian embedding, the metric is effectively that of a solution with an Abelian field. This was the only kind of regular solutions thought to exist in the Einstein-Yang-Mills theory, basically because the non-Abelian fields were expected to behave at infinity like the Abelian ones [2-4]. See also See refs. [5, 6] and references therein.

${ }^{2}$ More information on these black holes and the String Theory computation of their BH entropy can be found in ref. [14] and references therein.

${ }^{3}$ Technically, this family of black holes is a solution of the $\mathrm{SU}(2)$-gauged $\mathrm{ST}[2,6]$ model of $\mathcal{N}=1, d=5$ supergravity. This model and the solution-generating technique used to obtain the black-hole family is described in full detail in an appendix of ref. [16].
} 
low-energy effective field theory of the Heterotic Superstring. Our solution is an exact supergravity solution but, clearly, the issue of $\alpha^{\prime}$ corrections needs to be addressed. As we show in appendix A, the supergravity solution we are studying is also good to order $\alpha^{\prime}$ in Heterotic Superstring theory, but only in the near-horizon region and needs to be $\alpha^{\prime}$-corrected elsewhere. Finding these corrections is a problem that we will tackle in a forthcoming publication [17] and, in the meantime, one can work with the supergravity solution within the limits we just mentioned. In particular, the supergravity solution should be enough to characterize the different branes the black hole is "made of".

Back to the non-Abelian hair puzzle, in this case at least, the solution lies in the correct interpretation of the different charges that characterize the black hole. As we have shown in ref. [16], the charges that count the underlying String-Theory objects are combinations of the naive ones. The correctly identified charges can be switched off one by one and, switching off those that count the objects that give rise to the Abelian charges (that is, setting to zero the number of D1s, D5s and the momentum) one is left with the object that produces the net non-Abelian field. In 5 dimensions, this object is a globally regular, horizonless gravitating instanton [16] which, when uplifted to 10-dimensional Heterotic Supergravity (the effective field theory of the Heterotic Superstring), is nothing but Strominger's gauge 5-brane [18]. ${ }^{4}$ In terms of these charges, as we will see, there is a non-Abelian contribution to the mass and the non-Abelian contribution to the entropy disappears, solving the puzzle.

This is a very important clue that we are going to apply to these solutions. In section 1 we are going to introduce them and rewrite them in terms of the charges that describe the underlying String Theory objects. In section 2 we are going to uplift them to 10-dimensional Heterotic Supergravity, a theory that has non-Abelian vector fields in 10 dimensions, and, in section 3 we will reinterpret the solution in terms of intersections of fundamental strings, solitonic 5-branes and gauge 5-branes, plus momentum along the strings, and we will dualize it into a solution of Type-I Supergravity (the effective field theory of Type-I Superstring Theory) [20-22] with D-strings, momentum, D5-branes and "gauge D5-branes", the duals of the gauge 5-branes, also referred to as D5-branes dissolved into the D9 branes. Then, in section 4 we discuss how this brane configuration leads to the same entropy as the Abelian one, pointing to directions for future work. Finally, in appendix A we discuss the validity of our solution of 10-dimensional Heterotic Supergravity as a solution of the Heterotic Superstring taking into account $\alpha^{\prime}$ corrections.

\section{5-dimensional non-Abelian black holes}

We consider the $\mathrm{SU}(2)$-gauged $\mathrm{ST}[2,6]$ model of $\mathcal{N}=1, d=5$ supergravity, which can be obtained from $d=10$ Heterotic Supergravity by compactification on $T^{5}$ followed by a truncation. This is most conveniently done in two stages: first, compactification on $T^{4}$ followed by a truncation to $\mathcal{N}=(2,0), d=6$ supergravity coupled to a tensor multiplet and a triplet of $\mathrm{SU}(2)$ vectors and, second, further compactification on $S^{1}$. The first stage

\footnotetext{
${ }^{4}$ For recent work on Abelian black-hole solutions of Heterotic Supergravity (with $R^{2}$ terms, the HullStrominger system) see ref. [19] and references therein.
} 
is almost trivial: all the 6-dimensional fields are identical (up to rescalings) to the first 6 components of the 10-dimensional ones. The second stage is described in detail in ref. [23].

This model is determined by the symmetric tensor $C_{0 x y}=\frac{1}{6} \eta_{x y}$, with $x, y=1,2, A$, $A, B, \ldots=3,4,5$ and $\eta_{x y}=(+,-,-,-,-) .{ }^{5}$ The $A, B, \ldots$ are adjoint $\mathrm{SU}(2)$ indices. The bosonic content of this model consists of the metric $g_{\mu \nu}, 3$ Abelian vectors, $A^{0}, A^{1}$ and $A^{2}$ a triplet of $\mathrm{SU}(2)$ vectors $A^{A}$, and 5 scalars which we choose as $\phi, k$ and $\ell^{A}$ where $\phi$ can be directly identified with the 10-dimensional heterotic dilaton and $k$ is the Kaluza-Klein scalar of the last compactification from $d=6$ to $d=5$.

A particularly simple family of non-Abelian black-hole solutions of $\mathcal{N}=1, d=5$ supergravity can be constructed by adding a BPST instanton to the standard 3-charge solution $[11,16,24]$. The family of solutions is determined by 3 harmonic functions $L_{0, \pm}$ which depend on three constants $B_{0, \pm}$ satisfying $\frac{27}{2} B_{0} B_{+} B_{-}=1$ and three independent charges $q_{0, \pm}$

$$
L_{0, \pm}=B_{0, \pm}+q_{0, \pm} / \rho^{2}
$$

and a non-Abelian contribution that depends on the 5-dimensional gauge coupling constant $g$ and on the instanton scale $\kappa$

$$
\Phi^{2} \equiv \frac{2 \kappa^{4}}{3 g^{2} \rho^{4}\left(\rho^{2}+\kappa^{2}\right)^{2}}
$$

The non-Abelian contribution appears combined with the harmonic function $L_{0}$ as follows:

$$
\tilde{L}_{0} \equiv L_{0}-\frac{1}{3} \rho^{2} \Phi^{2}
$$

and, since it goes like $1 / \rho^{6}$ at spatial infinity while $L_{0}$ goes like $B_{0}+q_{0} / \rho^{2}$, it is not expected to contribute to the mass. However, both the Abelian and non-Abelian contributions diverge like $1 / \rho^{2}$ near the horizon at $\rho=0$, and, naively, one expects both of them to contribute to the entropy. This can be manifest by rewriting $\tilde{L}_{0}$ as

$$
\tilde{L}_{0}=B_{0}+\left(q_{0}-\frac{2}{9 g^{2}}\right) \frac{1}{\rho^{2}}+\frac{2}{9 g^{2}} \frac{\rho^{2}+2 \kappa^{2}}{\left(\rho^{2}+\kappa^{2}\right)^{2}},
$$

where we have combined Abelian and non-Abelian $1 / \rho^{2}$ terms in $\tilde{L}_{0}$, leaving a purely nonAbelian contribution which is finite at $\rho=0$. As in ref. [16], we will call $\tilde{q}_{0} \equiv q_{0}-\frac{2}{9 g^{2}}$ the coefficient of the $1 / \rho^{2}$ term.

The constants $B_{0, \pm}$ are related to the moduli i.e. the values of the 2 scalars at infinity, ${ }^{6}$ as follows

$$
B_{0}=\frac{1}{3} e^{\phi_{\infty}} k_{\infty}^{-2 / 3}, \quad B_{-}=\frac{2}{3} e^{-\phi_{\infty}} k_{\infty}^{-2 / 3}, \quad B_{+}=\frac{1}{3} k_{\infty}^{4 / 3} .
$$

Is is convenient to use the functions $\tilde{\mathcal{Z}}_{0} \equiv \tilde{L}_{0} / B_{0}$ and $\mathcal{Z}_{ \pm} \equiv L_{ \pm} / B_{ \pm}$and the charges $\tilde{\mathcal{Q}}_{0} \equiv \tilde{q}_{0} / B_{0}=\left(q_{0}-\frac{2}{9 g^{2}}\right) / B_{0}$ and $\mathcal{Q}_{ \pm} \equiv q_{ \pm} / B_{ \pm}$.

\footnotetext{
${ }^{5} \mathrm{~A}$ more detailed description of this model can be found in appendix A of ref. [16], for instance.

${ }^{6}$ We will relate the charges to the numbers of branes in $d=10$ after embedding the solution in Heterotic Supergravity.
} 
It is also convenient to transform the BPST instanton field from the gauge used in refs. $[11,12]$ to one in which the 10 -dimensional solution will be easier to recognize: ${ }^{7,8}$

$$
A_{R}^{A}=\frac{1}{g} \frac{\kappa^{2}}{\left(\kappa^{2}+\rho^{2}\right)} v_{R}^{A} \longrightarrow A_{L}^{A}=-\frac{1}{g} \frac{\rho^{2}}{\left(\kappa^{2}+\rho^{2}\right)} v_{L}^{A} .
$$

The vector field strength is, evidently, the same, but the Chern-Simons term is not and this difference will also affect the 10-dimensional 2-form.

After all these transformations, the active fields of the solutions are ${ }^{9}$

$$
\begin{aligned}
d s^{2} & =f^{2} d t^{2}-f^{-1}\left(d \rho^{2}+\rho^{2} d \Omega_{(3)}^{2}\right), & & \\
A^{0} & =-\sqrt{3} e^{-\phi_{\infty}} k_{\infty}^{2 / 3} \frac{d t}{\tilde{\mathcal{Z}}_{0}}, & A^{1}+A^{2} & =-2 \sqrt{3} k_{\infty}^{-4 / 3} \frac{d t}{\mathcal{Z}_{+}}, \\
A^{A} & =-\frac{1}{g} \frac{\rho^{2}}{\left(\kappa^{2}+\rho^{2}\right)} v_{L}^{A}, & A^{1}-A^{2} & =-\sqrt{3} e^{\phi_{\infty}} k_{\infty}^{2 / 3} \frac{d t}{\mathcal{Z}_{-}}, \\
e^{2 \phi} & =e^{2 \phi_{\infty}} \frac{\tilde{\mathcal{Z}}_{0}}{\mathcal{Z}_{-}}, & k & =k_{\infty}\left(f \mathcal{Z}_{+}\right)^{3 / 4},
\end{aligned}
$$

where the metric function $f$ is given by

$$
f^{-3}=\tilde{\mathcal{Z}}_{0} \mathcal{Z}_{+} \mathcal{Z}_{-}
$$

and the $\mathcal{Z}$ functions take the form

$$
\begin{aligned}
& \tilde{\mathcal{Z}}_{0}=1+\frac{\tilde{\mathcal{Q}}_{0}}{\rho^{2}}+\frac{2 e^{-\phi_{\infty}} k_{\infty}^{2 / 3}}{3 g^{2}} \frac{\rho^{2}+2 \kappa^{2}}{\left(\rho^{2}+\kappa^{2}\right)^{2}} \\
& \mathcal{Z}_{ \pm}=1+\frac{\mathcal{Q}_{ \pm}}{\rho^{2}}
\end{aligned}
$$

The mass and entropy of this family of black-hole solutions take the form

$$
\begin{aligned}
M & =\frac{\pi}{4 G_{N}^{(5)}}\left[\tilde{\mathcal{Q}}_{0}+\frac{2 e^{-\phi_{\infty}} k_{\infty}^{2 / 3}}{3 g^{2}}+\mathcal{Q}_{+}+\mathcal{Q}_{-}\right], \\
S & =\frac{\pi^{2}}{2 G_{N}^{(5)}} \sqrt{\tilde{\mathcal{Q}}_{0} \mathcal{Q}_{+} \mathcal{Q}_{-}} .
\end{aligned}
$$

Using the charge $\tilde{\mathcal{Q}}_{0}$ instead of $\mathcal{Q}_{0} \equiv q_{0} / B_{0}$, and assuming that $\tilde{\mathcal{Q}}_{0}$ is not related to the non-Abelian fields, the mass contains a net $\mathcal{O}\left(1 / g^{2}\right)$ contribution from the instanton while the entropy does not, against the naive expectations exposed above. We are going to

\footnotetext{
${ }^{7}$ The reason why this gauge was not used in refs. $[11,12]$ is that, in it, the gauge field cannot be consistently reduced following Kronheimer.

${ }^{8}$ Our conventions for the $\mathrm{SU}(2)$ gauge fields are slightly different from the ones used in refs. [11, 12]. Here the generators satisfy the algebra $\left[T_{A}, T_{B}\right]=+\epsilon_{A B C} T_{C}$, the left-invariant Maurer-Cartan 1-forms are defined by $v_{L} \equiv-U^{-1} d U$ and the right-invariant ones by $v_{R} \equiv-d U U^{-1}$. The gauge field strength is defined by $F=d A+g A \wedge A$.

${ }^{9}$ Since we are going to use hats to denote 10-dimensional fields, we have removed the hats that we use in our notation for the metric function $f$.
} 
argue that, indeed, $\tilde{\mathcal{Q}}_{0}$ is a charge completely unrelated to the non-Abelian vector fields, showing that it counts the number of neutral 5-branes (also known as solitonic or NSNS 5-branes) while $\mathcal{Q}_{-}$and $\mathcal{Q}_{+}$count, respectively, the number of fundamental strings and the momentum along them. Setting these three charges to zero we are left with the only nonAbelian component of this solution which is the globally regular and horizonless gravitating Yang-Mills instanton that we have found in ref. [16], showing that it is nothing but the dimensional reduction of Strominger's gauge 5-brane [18].

In ref. [16] we have argued that the gravitating Yang-mills instanton (or the gauge 5-branes) should not contribute to the entropy while, obviously, it must contribute to the total mass of black-hole solutions, just as the global monopole does in 4 dimensions [25, 26]. The above mass and entropy formulae reflect this fact.

\section{$2 \quad$ Embedding in $d=10$ Heterotic Supergravity}

As a first step towards embedding the 5-dimensional supergravity black hole solution into Heterotic Superstring theory, we are going to embed it in 10-dimensional Heterotic Supergravity $(\mathcal{N}=1, d=10$ supergravity coupled to vector supermultiplets), with just an $\mathrm{SU}(2)$ triplet of gauge fields. Since the 5-dimensional theory we start from does not have any terms of higher order in curvatures, we do not consider this kind of terms in the 10-dimensional theory. Observe, however, that the gauge fields occur at first order in $\alpha^{\prime}$ and, since our non-Abelian solution has non-trivial vector fields, in order to be consistent we are forced to study its validity as solution of Heterotic Superstring theory to first order in $\alpha^{\prime}$. At this order there are other terms in the action and we are going to study their relevance for this solution in appendix A.

First of all, we are going to show how the reduction and truncation of the bosonic sector of the 10-dimensional Heterotic Supergravity with a SU(2) triplet of gauge fields leads to the $\mathrm{SU}(2)$-gauged $\mathrm{ST}[2,6]$ model of $\mathcal{N}=1, d=5$ supergravity we are working with.

The action of Heterotic Supergravity in the string frame, including only a SU(2) triplet of vector fields, is

$$
\hat{S}=\frac{g_{s}^{2}}{16 \pi G_{N}^{(10)}} \int d^{10} x \sqrt{|\hat{g}|} e^{-2 \hat{\phi}}\left[\hat{R}-4(\partial \hat{\phi})^{2}+\frac{1}{2 \cdot 3 !} \hat{H}^{2}-\alpha^{\prime} \hat{F}^{A} \hat{F}^{A}\right],
$$

where the field strengths are defined as

$$
\begin{aligned}
\hat{F}^{A} & =d \hat{A}^{A}+\frac{1}{2} \epsilon^{A B C} \hat{A}^{B} \wedge \hat{A}^{C}, \\
\hat{H} & =d \hat{B}+2 \alpha^{\prime} \hat{\omega}_{\mathrm{YM}},
\end{aligned}
$$

and $\omega_{\mathrm{YM}}$ is the Chern-Simons 3-form

$$
\omega_{\mathrm{YM}} \equiv \hat{F}^{A} \wedge \hat{A}^{A}-\frac{1}{3 !} \epsilon^{A B C} \hat{A}^{A} \wedge \hat{A}^{B} \wedge \hat{A}^{C}, \quad d \omega_{\mathrm{YM}}=\hat{F}^{A} \wedge \hat{F}^{A} .
$$

In the above expressions, $\alpha^{\prime}$, the Regge slope, is related to the string length $\ell_{s}$ by $\alpha^{\prime}=\ell_{s}^{2}$, and $g_{s}$, the string coupling constant, is the value of the exponential of the dilaton at infinity: 
$g_{s}=e^{\phi_{\infty}}$ in asymptotically-flat configurations. The somewhat unconventional factor of $g_{s}^{2}$ in front of the action ensures that, after a rescaling from the string frame to the modified Einstein frame defined in ref. [27] with powers of $e^{\phi-\phi_{\infty}}$, the action has the standard normalization factor $\left(16 \pi G_{N}^{(10)}\right)^{-1}$. The 10 -dimensional Newton constant is given by

$$
G_{N}^{(10)}=8 \pi^{6} g_{s}^{2} \ell_{s}^{8}
$$

If we compactify this theory on $T^{4}$, it is not difficult to see that truncating all the components of the fields with indices in the internal coordinates $y^{i}, i=1, \cdots, 4$, is a consistent truncation. The resulting 6-dimensional action and field strengths have exactly the same form as the 10-dimensional ones, although the action carries an extra factor $\left(2 \pi \ell_{s}\right)^{4}$ which is the volume of the $T^{4}$ :

$$
\hat{S}=\frac{\left(2 \pi \ell_{s}\right)^{4} g_{s}^{2}}{16 \pi G_{N}^{(10)}} \int d^{6} x \sqrt{|\hat{g}|} e^{-2 \hat{\phi}}\left[\hat{R}-4(\partial \hat{\phi})^{2}+\frac{1}{2 \cdot 3 !} \hat{H}^{2}-\alpha^{\prime} \hat{F}^{A} \hat{F}^{A}\right] .
$$

The 6-dimensional modified Einstein metric $\hat{g}_{E} \hat{\mu} \hat{\nu}$ is related to the 6 -dimensional string metric $\hat{g}_{\hat{\mu} \hat{\nu}}$ by

$$
\hat{g}_{\hat{\mu} \hat{\nu}}=g_{s}^{-1} e^{\hat{\phi}} \hat{g}_{E \hat{\mu} \hat{\nu}}
$$

and, in this frame, the action takes the form

$$
\hat{S}=\frac{\left(2 \pi \ell_{s}\right)^{4}}{16 \pi G_{N}^{(10)}} \int d^{6} x \sqrt{\left|\hat{g}_{E}\right|}\left[\hat{R}_{E}+(\partial \hat{\phi})^{2}+\frac{1}{2 \cdot 3 !} g_{s}^{2} e^{-2 \hat{\phi}} \hat{H}^{2}-\alpha^{\prime} g_{s} e^{-\hat{\phi}} \hat{F}^{A} \hat{F}^{A}\right]
$$

which coincides exactly with the action of the theory of gauged $\mathcal{N}=(2,0), d=6$ supergravity that we called $\mathcal{N}=2 A$ in ref. [23] upon the redefinitions

$$
\hat{\phi}=-\tilde{\varphi} / \sqrt{2}, \quad g_{s} \hat{H} / 2=\tilde{H}, \quad \sqrt{g_{s} \alpha^{\prime}} \hat{F}^{A}=\tilde{F}^{A}
$$

which lead to the introduction of the 6-dimensional Yang-Mills coupling constant $g_{6}=\left(g_{s} \alpha^{\prime}\right)^{-1 / 2}$.

Further compactification of this theory on a circle leads to the $\mathrm{SU}(2)$-gauged $\mathrm{ST}[2,6]$ model of $\mathcal{N}=1, d=5$ supergravity we are working with, with Newton and Yang-Mills constants given by

$$
G_{N}^{(5)}=\frac{G_{N}^{(10)}}{(2 \pi)^{5} \ell_{s}^{4} R_{z}}=\frac{\pi g_{s}^{2} \ell_{s}^{4}}{4 R_{z}}, \quad \text { and } \quad g=\frac{g_{6} k_{\infty}^{1 / 3}}{\sqrt{12}}=\frac{R_{z}^{1 / 3}}{\sqrt{12 g_{s} \ell_{s}^{2}}} .
$$

This reduction was carried out in detail in ref. [23] and we can use its results, but we have to take into account that we have to rescale the 5-dimensional metric with the KaluzaKlein scalar $k$ divided by its asymptotic value, $k_{\infty}$ in order to preserve the normalization of asymptotically-flat metrics. This introduces an additional factor of $k_{\infty}^{1 / 3}$ in the relations between higher-dimensional fields and 5 -dimensional vector fields and an additional factor of $k_{\infty}^{2 / 3}$ in the relations between higher-dimensional fields and 5-dimensional 2-form fields. 
Combining the $k_{\infty}$-corrected rules given in ref. [23] to uplift 5-dimensional configurations to $d=6$ and the relations given above between 6 - and 10-dimensional fields in the string frame, we arrive to the following rules that allow us to uplift any solution of the $\mathrm{SU}(2)$-gauged $\mathrm{ST}[2,6]$ model of $\mathcal{N}=1, d=5$ supergravity to a solution of 10-dimensional Heterotic Supergravity preserving the normalization of the fields at spatial infinity:

$$
\begin{aligned}
d \hat{s}^{2} & =e^{\phi-\phi_{\infty}}\left[\left(k / k_{\infty}\right)^{-2 / 3} d s^{2}-k^{2} \mathcal{A}^{2}\right]-d y^{i} d y^{i}, \\
\hat{\phi} & =\phi \\
\hat{A}^{A} & =\frac{k_{\infty}^{1 / 3}}{\sqrt{12 g_{s} \alpha^{\prime}}} A^{A}+\frac{\ell^{A}}{\sqrt{\alpha^{\prime} g_{s}}} \mathcal{A}, \\
\hat{H} & =-\frac{k_{\infty}^{2 / 3}}{g_{s} \sqrt{3}} e^{2 \phi} k^{-4 / 3}{ }_{{ }_{(5)}} F^{0}+\frac{k_{\infty}^{1 / 3}}{g_{s} \sqrt{3}} \mathcal{A} \wedge \mathcal{F},
\end{aligned}
$$

where we have introduced the auxiliary fields

$$
\begin{array}{ll}
\mathcal{A} \equiv d z+\frac{k_{\infty}^{1 / 3}}{\sqrt{12}} A^{+}, & A^{+} \equiv A^{1}+A^{2}, \\
\mathcal{F} & \equiv F^{-}+\ell^{2} F^{+}+2 \ell^{A} F^{A} .
\end{array}
$$

Notice that the map gives us the 3 -form field strength $\hat{H}$, but not the 2 -form potential $\hat{B}$ because the process involves a dualization. Therefore $\hat{B}$ must be obtained from (2.3) once the field strengths $\hat{H}$ and $\hat{F}^{A}$ have been computed.

\section{String theory interpretation}

Using the uplifting formulae of the previous section, and defining the coordinate $u=k_{\infty} z$ (whose period is $2 \pi R_{z}$ ) we get the following solution of $d=10$ Heterotic Supergravity

$$
\begin{aligned}
d \hat{s}^{2} & =\frac{2}{\mathcal{Z}_{-}} d u\left(d v-\frac{1}{2} \mathcal{Z}_{+} d u\right)-\tilde{\mathcal{Z}}_{0}\left(d \rho^{2}+\rho^{2} d \Omega_{(3)}^{2}\right)-d y^{i} d y^{i}, \quad i=1,2,3,4 \\
\hat{B} & =-\frac{1}{\mathcal{Z}_{-}} d v \wedge d u-\frac{1}{4} \tilde{\mathcal{Q}}_{0} \cos \theta d \psi \wedge d \phi \\
\hat{A}^{A} & =-\frac{\rho^{2}}{\left(\kappa^{2}+\rho^{2}\right)} v_{L}^{A} \\
e^{-2 \hat{\phi}} & =e^{-2 \hat{\phi}_{\infty}} \frac{\mathcal{Z}_{-}}{\tilde{\mathcal{Z}}_{0}}
\end{aligned}
$$

where $\tilde{\mathcal{Z}}_{0}$ and $\mathcal{Z}_{ \pm}$are given in eqs. (1.9). In terms of the stringy constants, $\tilde{\mathcal{Z}}_{0}$ is given by

$$
\tilde{\mathcal{Z}}_{0}=1+\frac{\tilde{\mathcal{Q}}_{0}}{\rho^{2}}+8 \alpha^{\prime} \frac{\rho^{2}+2 \kappa^{2}}{\left(\rho^{2}+\kappa^{2}\right)^{2}} .
$$

As shown in appendix A, for $\tilde{Q}_{0} \gg \kappa^{2}$ this is a good solution of the Heterotic Superstring effective action to order $\alpha^{\prime}$ in the near-horizon $(\rho \rightarrow)$ region. This is enough for our purposes. 
Eq. (3.2) shows that the charge $\tilde{\mathcal{Q}}_{0}$ which is the coefficient of the $1 / \rho^{2}$ term is probably associated to neutral (or solitonic or NSNS) 5-branes [28] while the last term should be associated to gauge 5-branes. We are first going to discuss this point in more detail.

We start by noticing that, in absence of the Yang-Mills instanton, this supergravity solution is the one found in refs. [29, 30] which describes solitonic 5-branes wrapped on $T^{5}$, and fundamental strings wrapped around one cycle of the $T^{5}$ with momentum along the same direction.

Let us consider the coupling of $N_{S 5}$ solitonic 5-branes lying in the directions $\frac{1}{2}(u+v), y^{1}, \cdots, y^{4}$, to the Heterotic Supergravity action given in eq. (2.1). Since the effective action of the solitonic 5-branes is written in terms of the NSNS 6-form $\tilde{B}$, we must first rewrite the action in terms of that field. It is convenient to use the language of differential forms, so the action eq. (2.1) takes the form

$$
\hat{S}=\frac{g_{s}^{2}}{16 \pi G_{N}^{(10)}} \int e^{-2 \hat{\phi}}\left[\star \hat{R}-4 d \hat{\phi} \wedge \star d \hat{\phi}+\frac{1}{2} \hat{H} \wedge \star \hat{H}+2 \alpha^{\prime} \hat{F}^{A} \wedge \star \hat{F}^{A}\right],
$$

and, after dualization $\star e^{-2 \hat{\phi}} \hat{H}=\hat{\tilde{H}} \equiv d \hat{\tilde{B}}$

$$
\begin{aligned}
\hat{S}=\frac{g_{s}^{2}}{16 \pi G_{N}^{(10)}} \int\{ & e^{-2 \hat{\phi}}\left[\star \hat{R}-4 d \hat{\phi} \wedge \star d \hat{\phi}+2 \alpha^{\prime} \hat{F}^{A} \wedge \star \hat{F}^{A}\right] \\
& \left.+\frac{1}{2} e^{2 \hat{\phi}} \hat{\tilde{H}} \wedge \star \hat{\tilde{H}}+2 \alpha^{\prime} \hat{\tilde{B}} \wedge \hat{F}^{A} \wedge \hat{F}^{A}\right\} .
\end{aligned}
$$

The 6-form will couple to the Wess-Zumino term in the effective action of $N_{S 5}$ coincident solitonic 5-branes via its pullback over the worldvolume

$$
N_{S 5} T_{S 5} g_{s}^{2} \int \phi_{*} \hat{\tilde{B}}, \quad \text { where } \quad T_{S 5}=\frac{1}{\left(2 \pi \ell_{s}\right)^{5} \ell_{s} g_{s}^{2}},
$$

and the 6 -form equation of motion is

$$
\frac{g_{s}^{2}}{16 \pi G_{N}^{(10)}}\left\{d\left(\star e^{2 \hat{\phi}} \hat{\tilde{H}}\right)-2 \alpha^{\prime} \hat{F}^{A} \wedge \hat{F}^{A}\right\}=g_{s}^{2} N_{S 5} T_{S 5} \star_{(4)} \delta^{(4)}(\rho),
$$

where $\star_{(4)} \delta^{(4)}(\rho)$ is a 4 -form in the 5-branes' transverse space whose integral gives 1 .

Integrating both sides of this equation over the transverse space ${ }^{10}$ we get

$$
\tilde{\mathcal{Q}}_{0}=\mathcal{Q}_{0}-8 \alpha^{\prime}=\ell_{s}^{2} N_{S 5}
$$

which confirms that $\tilde{\mathcal{Q}}_{0}=\mathcal{Q}_{0}-8 \alpha^{\prime} n$, where $n$ would the instanton number in more general configurations counts solitonic (neutral) 5-branes. The number of gauge 5-branes $N_{G 5}$

\footnotetext{
${ }^{10}$ We replace $\star e^{2 \hat{\phi}} \hat{\tilde{H}}$ by $\hat{H}$ for simplicity and use Stokes' theorem in the first term. For the second term we have

$$
\frac{1}{16 \pi^{2}} \int_{\mathbb{R}^{4}} \hat{F}^{A} \wedge \hat{F}^{A}=1,
$$

the instanton number.
} 
coincides with the instanton number $n$. Thus, we conclude that the parameter $\mathcal{Q}_{0}$ of the solution is

$$
\mathcal{Q}_{0}=\ell_{s}^{2}\left(N_{S 5}+8 N_{G 5}\right)
$$

The function $\mathcal{Z}_{-}$is clearly associated to 10 -dimensional fundamental strings wrapped around the coordinate $\frac{1}{2}(u-v)$. If we couple $N_{F 1}$ fundamental strings lying in the direction $\frac{1}{2}(u-v)$ we have

$$
T_{F 1} N_{F 1}=\frac{g_{s}^{2}}{16 \pi G_{N}^{(10)}} \int_{V^{8}} d\left(\star e^{-2 \hat{\phi}} \hat{H}\right), \quad \text { where } \quad T_{F 1}=\frac{1}{2 \pi \alpha^{\prime}}
$$

where $V^{8}$ is the space transverse to worldsheet parametrized by $u$ and $v$, whose boundary is the product $T_{4} \times S_{\infty}^{3}$. Using Stokes' theorem and the value of volume of $T^{4}\left(2 \pi \ell_{S}\right)^{4}$, we get

$$
\mathcal{Q}_{-}=\ell_{s}^{2} g_{s}^{2} N_{F 1}
$$

Finally, the function $\mathcal{Z}_{+}$is associated to a gravitational wave moving in the compact direction $\frac{1}{2}(u-v)$ at the speed of light. The simplest way to compute its momentum is to T-dualize the solution along that direction. This operation interchanges winding number $\left(N_{F 1}\right)$ and momentum $\left(N_{W}\right)$ and, at the level of the solution, it interchanges the functions $\mathcal{Z}_{-}$and $\mathcal{Z}_{+}$or, equivalently, the constants $\mathcal{Q}_{-}$and $\mathcal{Q}_{+}$. Thus,

$$
\mathcal{Q}_{+}=\ell_{s}^{2} g_{s}^{\prime 2} N_{F 1}^{\prime}=\ell_{s}^{2}\left(g_{s} \ell_{s} / R_{z}\right)^{2} N_{W}=\frac{g_{s}^{2} \ell^{4}}{R_{z}^{2}} N_{W}
$$

where we have taken into account the transformation of the string coupling constant under T-duality.

We conclude that the fields that give rise to the 5-dimensional non-Abelian black hole in eq. (1.7), (1.8) and (1.9) correspond to those sourced by $N_{F 1}$ fundamental strings wrapped around the 6th dimension with $N_{W}$ units of momentum moving in the same direction and $N_{S 5}$ solitonic (neutral) and $N_{G 5}=1$ gauge 5 -branes wrapped around the 6 th direction and a $T^{4}$. In terms of these numbers, the black hole's mass and the entropy in eqs. (1.10) and (1.11) take the form

$$
\begin{aligned}
M & =\frac{R_{z}}{g_{s}^{2} \ell_{s}^{2}}\left(N_{S 5}+8 N_{G 5}\right)+\frac{R_{z}}{\ell_{s}^{2}} N_{F 1}+\frac{1}{R_{z}} N_{W}, \\
S & =2 \pi \sqrt{N_{F 1} N_{W} N_{S 5}} .
\end{aligned}
$$

Unfortunately, the dynamics of String Theory in the background of non-perturbative objects such as solitonic and gauge 5-branes is not as well understood as its dynamics in the background of D-branes. Therefore, it is convenient to perform a strong-weak coupling 
Heterotic-Type-I duality transformation [20-22] which acts on the fields as follows: ${ }^{11,12}$

$$
\hat{g}_{\hat{\mu} \hat{\nu}}=e^{-\left(\hat{\varphi}-\hat{\varphi}_{\infty}\right)} \hat{\jmath}_{\hat{\mu} \hat{\nu}}, \quad \hat{\phi}=-\hat{\varphi}, \quad \hat{C}_{\hat{\mu} \hat{\nu}}^{(2)}=e^{-\hat{\varphi}_{\infty}} \hat{B}_{\hat{\mu} \hat{\nu}} \quad \hat{A}_{\hat{\mu}}^{A}=g_{I}^{1 / 2} \hat{\mathcal{A}}_{\hat{\mu}}^{A},
$$

where $g_{I} \equiv e^{\hat{\varphi}_{\infty}}$ is the Type-I string coupling constant. These transformations lead to the Type-I supergravity action

$$
g_{I}^{-4} \hat{S}_{I}=\frac{g_{I}^{2}}{16 \pi G_{N, I}^{(10)}} \int\left\{e^{-2 \hat{\varphi}}[\star \hat{R}-4 d \hat{\varphi} \wedge \star d \hat{\varphi}]+\frac{1}{2} \hat{G}^{(3)} \wedge \star \hat{G}^{(3)}+2 \alpha^{\prime} e^{-\hat{\varphi}} \hat{\mathcal{F}}^{A} \wedge \star \hat{\mathcal{F}}^{A}\right\},
$$

and our solution takes the form

$$
\begin{aligned}
d \hat{s}_{I}^{2} & =\frac{2}{\sqrt{\tilde{\mathcal{Z}}_{0} \mathcal{Z}_{-}}} d u\left(d v-\frac{1}{2} \mathcal{Z}_{+} d u\right)-\sqrt{\tilde{\mathcal{Z}}_{0} \mathcal{Z}_{-}}\left(d \rho^{2}+\rho^{2} d \Omega_{(3)}^{2}\right)-\sqrt{\frac{\mathcal{Z}_{-}}{\tilde{\mathcal{Z}}_{0}}} d y^{i} d y^{i}, \\
\hat{C}^{(2)} & =-\frac{e^{-\hat{\varphi}_{\infty}}}{\mathcal{Z}_{-}} d v \wedge d u-\frac{e^{-\hat{\varphi}_{\infty}}}{4} \tilde{\mathcal{Q}}_{0} \cos \theta d \psi \wedge d \phi \\
\hat{\mathcal{A}}^{A} & =-e^{-\hat{\varphi}_{\infty} / 2} \frac{\rho^{2}}{\left(\kappa^{2}+\rho^{2}\right)} v_{L}^{A} \\
e^{-2 \hat{\varphi}} & =e^{-2 \hat{\varphi}_{\infty}} \frac{\tilde{\mathcal{Z}}_{0}}{\mathcal{Z}_{-}}
\end{aligned}
$$

In agreement with the fact that under Heterotic/Type-I duality fundamental strings and solitonic 5-branes transform into D1- and D5-branes, respectively, gravitational waves remain gravitational waves with the same momentum, this solution describes the fields produced by a D5-brane intersecting a D1-brane in the $z$ direction with a wave propagating along that direction. The Yang-Mills instanton is a non-perturbative configuration of the non-Abelian Born-Infeld field that occurs in the worldvolume of the parallel D9-branes that

\footnotetext{
${ }^{11}$ These are the transformations that preserve the normalization of the string metric at spatial infinity and lead to the correct normalization of the action of the Type-I theory. In particular, the rescaling of the gauge fields is required in order to reproduce correctly the term that appears in the expansion of the Born-Infeld action of the O9-D9-brane system (in the Abelian case). The effective worldvolume action of the D9-brane (Born-Infeld plus Wess-Zumino (WZ) terms) is

$$
\hat{S}_{D 9}=T_{D 9} g_{I} \int d \xi^{10} e^{-\hat{\varphi}} \sqrt{\operatorname{det}\left(\hat{\jmath}_{i j}+2 \pi \alpha^{\prime} \hat{\mathcal{F}}_{i j}\right)}+W Z
$$

where $g_{I}$ is the Type I string coupling constant. In the physical gauge, ignoring the cosmological constanttype term because it will be cancelled by the O9-planes, and using $T_{D 9}=\left[\left(2 \pi \ell_{s}\right)^{9} \ell_{s} g_{I}\right]^{-1}$ we get

$$
\hat{S}_{D 9} \sim \frac{g_{I}^{2}}{16 \pi G_{N, I}^{(10)}} \int d^{10} x \sqrt{|\hat{\jmath}|}\left[\alpha^{\prime} e^{-\hat{\varphi}} \hat{\mathcal{F}}^{2}\right]+W Z
$$

where, now, $16 \pi G_{N, I}^{(10)}=\left(2 \pi \ell_{s}\right)^{7} \ell_{s} g_{I}^{2}$. If we rewrite the Type-I supergravity action in terms of the RR 6-form $\hat{C}^{(6)}$, just as in the Heterotic case, we get a term $\hat{C}^{(6)} \wedge \hat{\mathcal{F}}^{A} \wedge \hat{\mathcal{F}}^{A}$. This term originates in the WZ term of the D9 effective action as well.

${ }^{12}$ The same procedure (a strong-weak coupling duality transformation within Type-IIB supergravity) was followed in ref. [13] to derive the D5D1W solution without non-Abelian fields from the solution in [29, 30] which can be embedded directly in the Type-IIB NSNS sector. The presence of non-Abelian vector fields suggests the route we have taken.
} 
give rise to the Type-I theory from the Type-IIB and sources D5-branes. Thus $N_{D 1}=N_{F 1}$, $N_{D 5}=N_{S 5}, N_{G D 5}=N_{G 5}$ and, in Type-I variables, the mass and entropy formulae take the form

$$
\begin{aligned}
M & =\frac{R_{z}}{g_{I} \ell_{s}^{2}}\left(N_{D 5}+8 N_{G D 5}\right)+\frac{R_{z}}{g_{I} \ell_{s}^{2}} N_{D 1}+\frac{1}{R_{z}} N_{W}, \\
S & =2 \pi \sqrt{N_{D 1} N_{D 5} N_{W}} .
\end{aligned}
$$

In absence of the instanton $\left(N_{G D 5}=0\right)$ this solution is identical to the one originally considered in ref. [13], which is itself very closely related to Strominger and Vafa's original model [31]. ${ }^{13}$ The same conditions (namely, that all the $N_{\mathrm{s}}$ are large and $N_{W} \gg N_{D 1, D 5}$ ) ensure that this solution describes at leading order in $\alpha^{\prime}$ (low curvature) and in $g_{s}$ (perturbative string theory) a good background for Type-IIB string theory.

\section{Discussion}

In the previous sections we have shown that the 5-dimensional supergravity black holes with 3 quantized Abelian charges $N_{D 1}, N_{D 5}, N_{W}$ and a non-Abelian instanton can be seen, up to dualities, as the fields associated to a 10-dimensional Type-IIB configuration with

1. An orientifold $\mathrm{O} 9_{+}$-plane and 16 D9-branes and their mirror images, that give rise to the Type-I superstring theory with gauge group $\mathrm{SO}(32)$ (see, e.g. [33] and references therein).

2. $N_{D 5}$ D5-branes wrapped around the 5th-9th directions and $N_{D 1}$ D-strings wrapped around the 5th direction with $N_{W}$ units of momentum along the 5 th direction. Open strings can end on these D-strings and D5-branes.

3. $N_{G D 5}=1$ "gauge D5-brane", sourced by an instanton field located in the 1st-4th dimensions, which are not compact. This brane, which is the dual of the heterotic gauge 5-brane is often referred to as a D5-brane "dissolved" into the spacetime-filling D9-branes and differs essentially from standard D5-branes because no strings can end on them.

Since the entropy of the D1D5W black holes can be understood as associated to the massless states associated to strings with one endpoint on a D1 and the other on a D5 (1-5 states) and this fact, as discussed in ref. [13] is unchanged by the presence of the D9-branes and $\mathrm{O}_{+}$-plane that defines the Type-I theory ${ }^{14}$ the microscopic interpretation of the entropy of these non-Abelian black holes must be the same as in the Abelian case and should give the same result at leading order. Observe that, as an intermediate step in the uplift of the solution to 10 dimensions one obtains a non-Abelian string solution in 6 dimensions with an $\mathrm{AdS}_{3} \times \mathrm{S}_{3}$ near-horizon geometry where the $\mathrm{AdS}_{3}$ radius only depends on 3 quantized Abelian charges $N_{D 1}, N_{D 5}, N_{W}$.

\footnotetext{
${ }^{13}$ See also refs. [14, 27, 32].

${ }^{14}$ The counting of states is, however, different since, as mentioned in ref. [13] one has to take into account the SU(2) degrees of freedom associated to the D5-brane of the Type-I string found in [34].
} 
It is important to stress that the correct identification of the charges and their meaning in terms of branes plays a crucial rôle to reach this conclusion as well as in solving the apparent non-Abelian hair problem explained in the Introduction. A more detailed study is, however, necessary to find corrections to the entropy. In particular, the $\alpha^{\prime}$ corrections to this solution in the asymptotic limit need to be determined (see the appendix).

In the last few years we have constructed non-Abelian static and rotating black-hole solutions in 4 and 5 dimensions [9-11, 25, 26, 35], as well as black-ring solutions [12] and microstate geometries [36] in 5 dimensions. All those constructed with "colored monopoles" in 4 dimensions and many of the 5-dimensional solutions exhibit non-Abelian hair which seems to contribute to the entropy or the angular momentum on the horizon but cannot be seen at infinity. Many of them can be uplifted to 10-dimensional Heterotic Supergravity and then dualized into Type-I Supergravity solutions and it is likely that the correct interpretation of the charges of those solutions is enough to understand the non-Abelian hair problem. Work in this direction is in progress.

\section{Acknowledgments}

TO would like to thank Ángel Uranga for very useful conversations. This work has been supported in part by the Spanish Government grants FPA2012-35043-C02-01 and FPA2015-66793-P (MINECO/FEDER, UE), the Centro de Excelencia Severo Ochoa Program grant SEV-2012-0249 and the Spanish Consolider-Ingenio 2010 program CPAN CSD2007-00042. The work of PAC was supported by a "la Caixa-Severo Ochoa" International pre-doctoral grant. The work of PFR was supported by the Severo Ochoa pre-doctoral grant SVP-2013-067903. TO wishes to thank M.M. Fernández for her permanent support.

\section{A The issue of $\alpha^{\prime}$ corrections}

As we have mentioned in the main body of the paper, the solution of 10-dimensional Heterotic Supergravity that we have obtained by uplifting the 5-dimensional non-Abelian supersymmetric black hole solution has non-trivial $\mathrm{SU}(2)$ gauge fields. These occur at first order in $\alpha^{\prime}$ in the low-energy Heterotic Superstring effective action together with other terms that we are going to describe following ref. [37], and which we have ignored. The purpose of this appendix is to study the relevance of the omitted terms for the solution at hands. Only if these are negligible with respect to those we have considered can the solution be considered a good solution of the Heterotic Superstring effective action to first order in $\alpha^{\prime}$.

At lowest order (zeroth) in $\alpha^{\prime}$, the Heterotic Superstring effective action is nothing by the action of pure $\mathcal{N}=1, d=10$ supergravity [38, 39]. The coupling to super-YangMills multiplets $[39,40]$ leads to the exactly supersymmetric Heterotic Supergravity theory described in section 2. From the point of view of the Heterotic Superstring effective action, the terms associated to the Yang-Mills fields are of higher order in $\alpha^{\prime}$ : their kinetic term occurs in the action eq. (2.1) at first order and their Chern-Simons 3-form $\omega_{\mathrm{YM}}$ occurs in the Kalb-Ramond 3-form field strength $\hat{H}$ at first order as well, eq. (2.3), modifying its 
Bianchi identity so that it takes the form

$$
d \hat{H}=2 \alpha^{\prime} \hat{F}^{A} \wedge \hat{F}^{A} .
$$

This correction in $\hat{H}$ introduces terms of second order in $\alpha^{\prime}$ in the action and in the Einstein equations but it is precisely this mixture of terms of different orders in $\alpha^{\prime}$ that is exactly supersymmetric and gauge invariant.

The existence of additional terms at first order in $\alpha^{\prime}$ in the Heterotic Superstring effective action is both a blessing, because it makes possible the Green-Schwarz anomalycancellation mechanism [41], and a curse because, once they are included, the action will only be supersymmetric and gauge-invariant to second order in $\alpha^{\prime}$ [42]. The addition of further $\alpha^{\prime}$ corrections only makes the action supersymmetric and gauge-invariant to higher order in $\alpha^{\prime}$ [37] and will not be considered here.

With the addition of the aforementioned missing terms, the Heterotic Superstring effective action takes the form

$$
\hat{S}=\frac{g_{s}^{2}}{16 \pi G_{N}^{(10)}} \int d^{10} x \sqrt{|\hat{g}|} e^{-2 \hat{\phi}}\left\{\hat{R}-4(\partial \hat{\phi})^{2}+\frac{1}{2 \cdot 3 !} \hat{H}^{2}-\alpha^{\prime}\left[\hat{F}^{A} \hat{F}^{A}+\hat{R}_{(-)} \hat{a}_{\hat{b}} \hat{R}_{(-)}{ }^{\hat{b}} \hat{a}\right]\right\}
$$

where

$$
\hat{R}_{(-)}{ }^{\hat{a}} \hat{b}_{(-)}{ }_{\hat{b}}^{\hat{a}}=\hat{R}_{(-) \hat{\mu} \hat{\nu}} \hat{a} \hat{b}_{(-)}^{\hat{\mu} \hat{\nu} \hat{b}} \hat{a} .
$$

Here $\hat{\Omega}_{(-)} \hat{a}_{\hat{b}}$ is one of the two torsionful spin connection 1 -forms that can be constructed by adding to the Levi-Civita spin connection $\hat{\omega}^{\hat{a} \hat{b}} 1$-form a torsion piece

$$
\hat{\Omega}_{( \pm)} \hat{a}_{\hat{b}}=\hat{\omega}^{\hat{a}}{ }_{\hat{b}} \pm \frac{1}{2} \hat{H}_{\hat{\mu}} \hat{a}_{\hat{b}} d x^{\hat{\mu}},
$$

whose curvature 2 -forms are defined by

$$
\hat{R}_{( \pm)}{ }^{\hat{a}} \hat{b}=d \hat{\Omega}_{( \pm)}{ }_{\hat{b}}^{\hat{b}}-\hat{\Omega}_{( \pm)}{ }^{\hat{a}} \hat{c} \wedge \hat{\Omega}_{( \pm)}{ }_{\hat{b}}^{\hat{b}} .
$$

The Kalb-Ramond field strength 3 -form is now given by

$$
\hat{H}=d \hat{B}+2 \alpha^{\prime}\left(\hat{\omega}_{\mathrm{YM}}+\hat{\omega}_{\mathrm{L}(-)}\right),
$$

where $\hat{\omega}_{\mathrm{L}( \pm)}$ is the Chern-Simons 3 -form of the torsionful spin connection $\hat{\Omega}_{( \pm)}$

$$
\hat{\omega}_{\mathrm{L}( \pm)}=d \hat{\Omega}_{( \pm)}{ }_{\hat{b}}^{\hat{b}} \wedge \hat{\Omega}_{( \pm)} \hat{b}_{\hat{a}}-\frac{2}{3} \hat{\Omega}_{( \pm)}{ }_{\hat{b}}^{\hat{b}} \wedge \hat{\Omega}_{( \pm)} \hat{b}_{\hat{c}} \wedge \hat{\Omega}_{( \pm)}{ }^{\hat{c}} \hat{a}
$$

and, correspondingly, its Bianchi identity becomes

$$
d \hat{H}=2 \alpha^{\prime}\left(\hat{F}^{A} \wedge \hat{F}^{A}+\hat{R}_{(-)} \hat{a}_{\hat{b}} \wedge \hat{R}_{(-)} \hat{b}_{\hat{a}}\right) .
$$

Written in this way, and besides the explicit ones, the action contains an infinite number of implicit $\alpha^{\prime}$ corrections which arise due to the recursive way in which $\hat{H}$ is defined, since it depends on the Chern-Simons form of $\hat{\Omega}_{(-)}$, which is defined in terms of $\hat{H}$. At the order at which we are working, it is enough to keep in the definitions of $\hat{\Omega}_{( \pm)}$only the terms of zeroth order in $\alpha^{\prime}$, that is

$$
\hat{\Omega}_{( \pm)}{ }_{\hat{b}}=\hat{\omega}^{\hat{a}}{ }_{\hat{b}} \pm \frac{1}{2} \hat{H}_{\hat{\mu}}^{(0)}{ }_{\hat{b}} d x^{\hat{\mu}}, \quad \text { where } \quad \hat{H}^{(0)} \equiv d \hat{B}
$$

and we will ignore all the $\alpha^{2}$ terms in the action eq. (A.2). 
Now, by plugging the solution eq. (3.1) into the equations of motion that follow from the action eq. (A.2) with the torsionful spin connection defined in the previous equation, we can study if they are satisfied to first order in $\alpha^{\prime}$.

Following the scheme explained in section 3 of ref. [43] the variations of the action with respect to the each field can be separated into variations with respect to explicit occurrence of the field in the action and variations with respect to the implicit occurrence via the torsionful spin connection. The former are the zeroth order equations plus terms proportional to the so-called " $\hat{T}$-tensors," which we will define shortly and are of order $\alpha^{\prime}$. According to the lemma proved in section 3 of ref. [37], the latter are of order $\alpha^{\prime}$ and proportional to the zeroth order equations of motion. Since the solution eq. (3.1) satisfies the zeroth order equations of motion up to terms of first order in $\alpha^{\prime}$, the implicit variations are of order $\alpha^{\prime 2}$ and can be ignored.

The conclusion is that it is enough to study the $\hat{T}$-tensor-corrected zeroth-order equations of motion. The $3 \hat{T}$-tensors that appear in the corrections are defined as

$$
\begin{aligned}
\hat{T}_{\hat{\mu} \hat{\nu} \hat{\rho} \hat{\sigma}} & \equiv \alpha^{\prime}\left[\hat{F}_{[\hat{\mu} \hat{\nu}}^{A} \hat{F}_{\hat{\rho} \hat{\sigma}]}^{A}+\hat{R}_{(-)[\hat{\mu} \hat{\nu} \mid} \hat{a}_{\hat{b}} \hat{R}_{(-) \mid \hat{\rho} \hat{\sigma}]} \hat{b}_{\hat{a}}\right], \\
\hat{T}_{\hat{\mu} \hat{\nu}} & \equiv \alpha^{\prime}\left[\hat{F}_{\hat{\mu} \hat{\rho}}{ }^{A} \hat{F}_{\hat{\nu}} \hat{\rho} A+\hat{R}_{(-) \hat{\mu} \hat{\rho} \hat{\hat{b}}} \hat{R}_{(-) \hat{\nu}}^{\hat{\rho} \hat{b}} \hat{a}\right] \\
\hat{T} & \equiv \hat{T}^{\hat{\mu}}{ }_{\hat{\mu}} .
\end{aligned}
$$

The 4 -form $\hat{T}$-tensor is the r.h.s. of the Bianchi identity of $\hat{H}$, the symmetric 2-index $\hat{T}$-tensor is the term that occurs in the Einstein equations and its trace occurs in the dilaton equation.

The Yang-Mills part of these tensors was included in the equations of motion of the Heterotic Supergravity eq. (2.1). Therefore, we just need to compute them and compare Lorentz curvature part with the Yang-Mills part. In other words, we need to compare the $\kappa$-dependent term with the rest, which should be much smaller. ${ }^{15}$

For the solution at hands, to $\mathcal{O}\left(\alpha^{2}\right)$, they are explicitly given by

$$
\begin{aligned}
\hat{T}^{(4)} \sim \alpha^{\prime}\left[\frac{\kappa^{4}}{\left(\kappa^{2}+\rho^{2}\right)^{4}}-\frac{\tilde{Q}_{0}^{2}}{\left(\tilde{Q}_{0}+\rho^{2}\right)^{4}}\right] d \rho \rho^{3} \wedge \sin \theta d \theta \wedge d \Psi \wedge d \phi, \\
\hat{T}_{u u}=-\alpha^{\prime} \frac{32 Q_{-} Q_{+} \rho^{4}\left[\tilde{Q}_{0}^{2}+\tilde{Q}_{0}\left(Q_{-}+3 \rho^{2}\right)+Q_{-}^{2}+3 Q_{-} \rho^{2}+3 \rho^{4}\right]}{\left(\tilde{Q}_{0}+\rho^{2}\right)^{4}\left(Q_{-}+\rho^{2}\right)^{4}}, \\
\hat{T}_{i j}=\alpha^{\prime} \delta_{i j} \frac{48 \rho^{2}}{\left(\tilde{Q}_{0}+\rho^{2}\right)^{5}}\left[\tilde{Q}_{0}^{2}-\frac{\kappa^{4}\left(\tilde{Q}_{0}+\rho^{2}\right)^{4}}{\left(\kappa^{2}+\rho^{2}\right)^{4}}\right] \\
\hat{T}=-\alpha^{\prime} \frac{192 \rho^{4}}{\left(\kappa^{2}+\rho^{2}\right)^{4}\left(\tilde{Q}_{0}+\rho^{2}\right)^{6}}\left[\kappa^{8} \tilde{Q}_{0}^{2}+4 \kappa^{6} \tilde{Q}_{0}^{2} \rho^{2}\right. \\
\left.-\kappa^{4}\left(\tilde{Q}_{0}^{4}+4 \tilde{Q}_{0}^{3} \rho^{2}+4 \tilde{Q}_{0} \rho^{6}+\rho^{8}\right)+4 \kappa^{2} \tilde{Q}_{0}^{2} \rho^{6}+\tilde{Q}_{0}^{2} \rho^{8}\right]
\end{aligned}
$$

\footnotetext{
${ }^{15} \mathrm{It}$ is worth stressing this point: since our starting point is not an exact solution of the action to zeroth order in $\alpha^{\prime}$, our goal is not to make the value of the $\hat{T}$-tensors as small as possible.
} 
where $\hat{T}^{(4)}=\frac{1}{4 !} \hat{T}_{\hat{\mu} \hat{\nu} \hat{\rho} \hat{\sigma}} d x^{\hat{\mu}} d x^{\hat{\nu}} d x^{\hat{\rho}} d x^{\hat{\sigma}}$ and $i, j=2,3,4,5$ label the 4 coordinates of the 5 branes worldvolume which are orthogonal to the wave.

Let us start by analyzing $\hat{T}^{(4)}$ : in the near-horizon region $\rho \rightarrow 0$ the leading term is

$$
\hat{T}^{(4)} \sim \alpha^{\prime}\left(\frac{1}{\kappa^{4}}-\frac{1}{\tilde{Q}_{0}^{2}}\right) d \rho \rho^{3} \wedge \sin \theta d \theta \wedge d \Psi \wedge d \phi .
$$

In this limit, the $\alpha^{\prime}$ corrections of our solution will be small if $\kappa^{-4} \gg \tilde{Q}_{0}^{-2}$, that is, if $\tilde{Q}_{0} \gg \kappa^{2}$ so the number of S5-branes is very large.

Asymptotically $(\rho \rightarrow \infty)$, the leading term is

$$
\hat{T}^{(4)} \sim \alpha^{\prime} \frac{\left(\kappa^{4}-\tilde{Q}_{0}^{2}\right)}{\rho^{8}} d \rho \rho^{3} \wedge \sin \theta d \theta \wedge d \Psi \wedge d \phi,
$$

and the absence of $\alpha^{\prime}$ corrections in this limit requires exactly the opposite to happen: $\tilde{Q}_{0} \ll \kappa^{2}$ and the number of S5-branes should be very small.

The analysis of the other tensors sheds identical results, indicating that we can only consider our solution a good solution of the Heterotic Superstring effective action in either the near-horizon $\rho \rightarrow 0$ region for $\tilde{Q}_{0} \gg \kappa^{2}$ or in the asymptotic $\rho \rightarrow 0$ region for $\tilde{Q}_{0} \ll \kappa^{2}$. In either case, the solution will have to be $\alpha^{\prime}$ corrected in the other region.

For the purpose of computing the entropy it is more convenient to take $\tilde{Q}_{0} \gg \kappa^{2}$ so that the near-horizon region is well described to order $\alpha^{\prime}$ in Heterotic Superstring effective action. The $\alpha^{\prime}$ corrections which are needed in the asymptotic limit will be determined and studied in a forthcoming publication [17].

Open Access. This article is distributed under the terms of the Creative Commons Attribution License (CC-BY 4.0), which permits any use, distribution and reproduction in any medium, provided the original author(s) and source are credited.

\section{References}

[1] J.A. Smoller, A.G. Wasserman, S.-T. Yau and J.B. McLeod, Smooth static solutions of the Einstein Yang-Mills equations, Commun. Math. Phys. 143 (1991) 115 [InSPIRE].

[2] D.V. Galtsov and A.A. Ershov, Nonabelian Baldness of Colored Black Holes, Phys. Lett. A 138 (1989) 160 [INSPIRE].

[3] A.A. Ershov and D.V. Galtsov, Nonexistence of regular monopoles and dyons in the SU(2) Einstein Yang-Mills theory, Phys. Lett. A 150 (1990) 159 [InSPIRE].

[4] P. Bizon and O.T. Popp, No hair theorem for spherical monopoles and dyons in SU(2) Einstein Yang-Mills theory, Class. Quant. Grav. 9 (1992) 193 [InSPIRE].

[5] M.S. Volkov and D.V. Gal'tsov, Gravitating nonAbelian solitons and black holes with Yang-Mills fields, Phys. Rept. 319 (1999) 1 [hep-th/9810070] [InSPIRE].

[6] D.V. Gal'tsov, Gravitating lumps, in proceedings of The 16th International Conference on General Relativity and Gravitation (GR16), N.T. Bishop and S.D. Maharaj eds., World Scientific, Singapore (2002) [hep-th/0112038]. 
[7] M.S. Volkov and D.V. Galtsov, NonAbelian Einstein Yang-Mills black holes, JETP Lett. 50 (1989) 346 [INSPIRE].

[8] P. Bizon, Colored black holes, Phys. Rev. Lett. 64 (1990) 2844 [INSPIRE].

[9] P. Meessen, Supersymmetric coloured/hairy black holes, Phys. Lett. B 665 (2008) 388 [arXiv:0803.0684] [INSPIRE].

[10] P. Meessen and T. Ortín, $\mathcal{N}=2$ super-EYM coloured black holes from defective Lax matrices, JHEP 04 (2015) 100 [arXiv: 1501.02078] [INSPIRE].

[11] P. Meessen, T. Ortín and P.F. Ramírez, Non-Abelian, supersymmetric black holes and strings in 5 dimensions, JHEP 03 (2016) 112 [arXiv: 1512.07131] [INSPIRE].

[12] T. Ortín and P.F. Ramírez, A non-Abelian Black Ring, Phys. Lett. B 760 (2016) 475 [arXiv: 1605.00005] [INSPIRE].

[13] C.G. Callan and J.M. Maldacena, D-brane approach to black hole quantum mechanics, Nucl. Phys. B 472 (1996) 591 [hep-th/9602043] [INSPIRE].

[14] J.R. David, G. Mandal and S.R. Wadia, Microscopic formulation of black holes in string theory, Phys. Rept. 369 (2002) 549 [hep-th/0203048] [inSPIRE].

[15] A.A. Belavin, A.M. Polyakov, A.S. Schwartz and Yu.S. Tyupkin, Pseudoparticle Solutions of the Yang-Mills Equations, Phys. Lett. B 59 (1975) 85 [INSPIRE].

[16] P.A. Cano, T. Ortín and P.F. Ramírez, A gravitating Yang-Mills instanton, JHEP 07 (2017) 011 [arXiv: 1704.00504] [INSPIRE].

[17] P.A. Cano, P. Meessen, T. Ortín and P.F. Ramírez, $\alpha^{\prime}$-corrected non-Abelian black holes in string theory, in preparation.

[18] A. Strominger, Heterotic solitons, Nucl. Phys. B 343 (1990) 167 [Erratum ibid. B 353 (1991) 565] [INSPIRE].

[19] N. Halmagyi, D. Israel and E.E. Svanes, The Abelian Heterotic Conifold, JHEP 07 (2016) 029 [arXiv: 1601.07561] [INSPIRE].

[20] A. Dabholkar, Ten-dimensional heterotic string as a soliton, Phys. Lett. B 357 (1995) 307 [hep-th/9506160] [INSPIRE].

[21] C.M. Hull, String-string duality in ten-dimensions, Phys. Lett. B 357 (1995) 545 [hep-th/9506194] [INSPIRE].

[22] J. Polchinski and E. Witten, Evidence for heterotic - type-I string duality, Nucl. Phys. B 460 (1996) 525 [hep-th/9510169] [rNSPIRE].

[23] P.A. Cano, T. Ortín and C. Santoli, Non-Abelian black string solutions of $\mathcal{N}=(2,0), D=6$ supergravity, JHEP 12 (2016) 112 [arXiv: 1607.02595] [INSPIRE].

[24] P. Bueno, P. Meessen, T. Ortín and P.F. Ramírez, Resolution of SU(2) monopole singularities by oxidation, Phys. Lett. B 746 (2015) 109 [arXiv:1503. 01044] [INSPIRE].

[25] M. Hübscher, P. Meessen, T. Ortín and S. Vaulà, $N=2$ Einstein-Yang-Mills's BPS solutions, JHEP 09 (2008) 099 [arXiv: 0806.1477] [INSPIRE].

[26] P. Bueno, P. Meessen, T. Ortín and P.F. Ramírez, $\mathcal{N}=2$ Einstein- Yang-Mills' static two-center solutions, JHEP 12 (2014) 093 [arXiv:1410.4160] [INSPIRE].

[27] J.M. Maldacena, Black holes in string theory, Ph.D. Thesis, Princeton University (1996). [hep-th/9607235]. 
[28] S.-J. Rey, The Confining Phase of Superstrings and Axionic Strings, Phys. Rev. D 43 (1991) 526 [INSPIRE].

[29] A.A. Tseytlin, Extreme dyonic black holes in string theory, Mod. Phys. Lett. A 11 (1996) 689 [hep-th/9601177] [INSPIRE].

[30] M. Cvetič and A.A. Tseytlin, Solitonic strings and BPS saturated dyonic black holes, Phys. Rev. D 53 (1996) 5619 [Erratum ibid. D 55 (1997) 3907] [hep-th/9512031] [InSPIRE].

[31] A. Strominger and C. Vafa, Microscopic origin of the Bekenstein-Hawking entropy, Phys. Lett. B 379 (1996) 99 [hep-th/9601029] [INSPIRE].

[32] A.W. Peet, TASI lectures on black holes in string theory, in proceedings of Strings, branes and gravity, TASI'99, Boulder, U.S.A. (1999). J.A. Harvey, S. Kachru and E. Silverstein eds., World Scientific (2001) [hep-th/0008241].

[33] C. Angelantonj and A. Sagnotti, Open strings, Phys. Rept. 371 (2002) 1 [hep-th/0204089] [INSPIRE].

[34] E. Witten, Small instantons in string theory, Nucl. Phys. B 460 (1996) 541 [hep-th/9511030] [INSPIRE].

[35] M. Hübscher, P. Meessen, T. Ortín and S. Vaulà, Supersymmetric $N=2$ Einstein-Yang-Mills monopoles and covariant attractors, Phys. Rev. D 78 (2008) 065031 [arXiv:0712.1530] [INSPIRE].

[36] P.F. Ramírez, Non-Abelian bubbles in microstate geometries, JHEP 11 (2016) 152 [arXiv: 1608.01330] [INSPIRE].

[37] E.A. Bergshoeff and M. de Roo, The Quartic Effective Action of the Heterotic String and Supersymmetry, Nucl. Phys. B 328 (1989) 439 [INSPIRE].

[38] A.H. Chamseddine, $N=4$ Supergravity Coupled to $N=4$ Matter, Nucl. Phys. B 185 (1981) 403 [INSPIRE].

[39] E. Bergshoeff, M. de Roo, B. de Wit and P. van Nieuwenhuizen, Ten-Dimensional Maxwell-Einstein Supergravity, Its Currents and the Issue of Its Auxiliary Fields, Nucl. Phys. B 195 (1982) 97 [INSPIRE].

[40] G.F. Chapline and N.S. Manton, Unification of Yang-Mills Theory and Supergravity in Ten-Dimensions, Phys. Lett. B 120 (1983) 105 [InSPIRE].

[41] M.B. Green and J.H. Schwarz, Anomaly Cancellation in Supersymmetric D = 10 Gauge Theory and Superstring Theory, Phys. Lett. B 149 (1984) 117 [inSPIRE].

[42] E. Bergshoeff and M. de Roo, Supersymmetric Chern-Simons Terms in Ten-dimensions, Phys. Lett. B 218 (1989) 210 [INSPIRE].

[43] E.A. Bergshoeff, R. Kallosh and T. Ortín, Supersymmetric string waves, Phys. Rev. D 47 (1993) 5444 [hep-th/9212030] [INSPIRE]. 